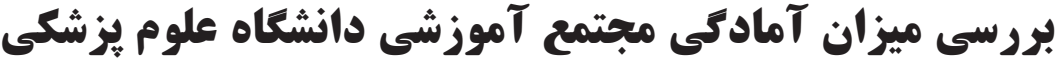

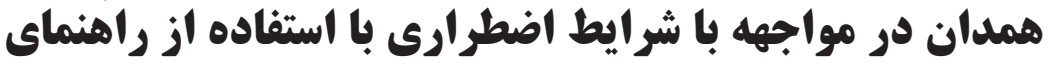

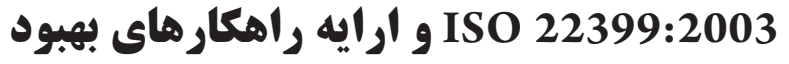

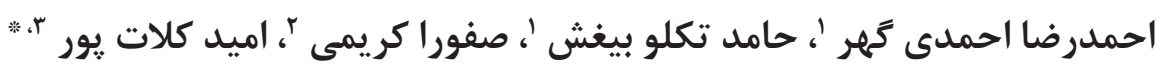

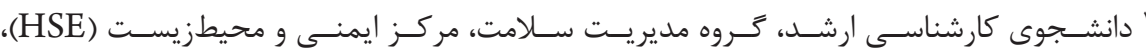

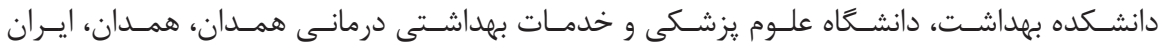

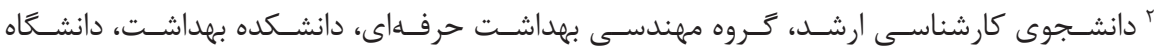

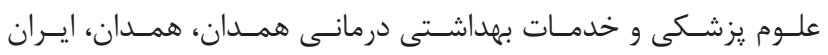

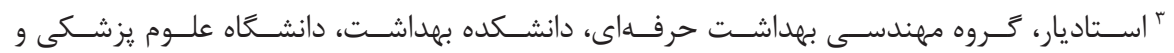

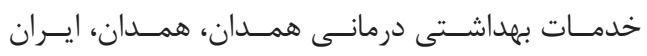

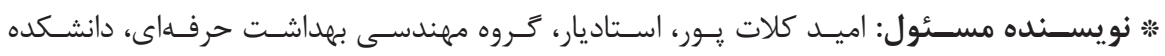

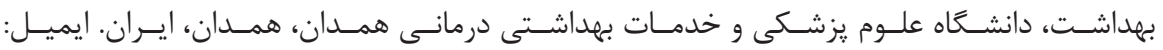

DOI: $10.21859 /$ johe- 03046

جكيده

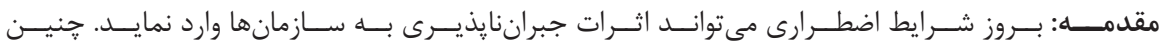

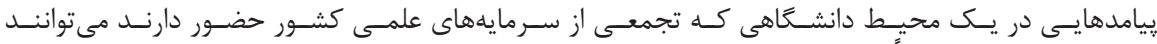

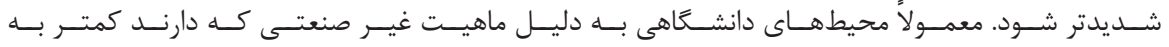

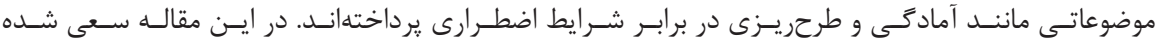

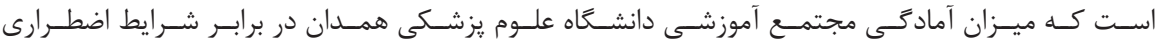

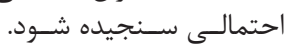

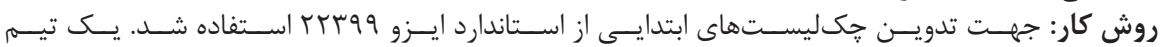

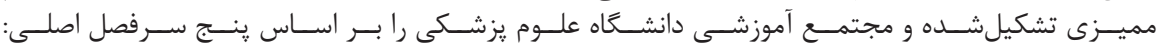

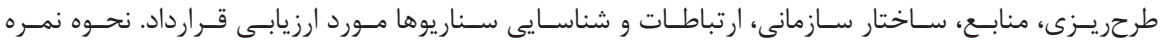

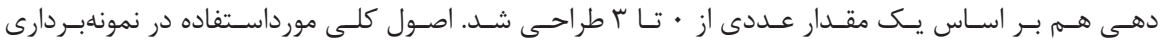

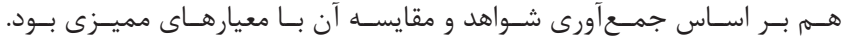

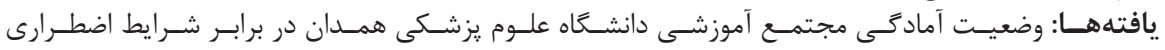

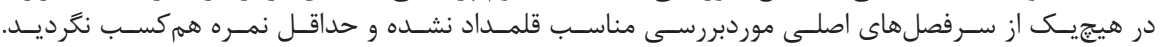

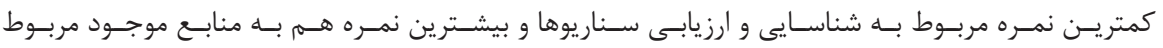

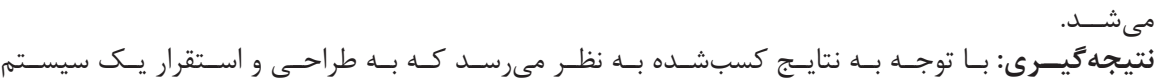

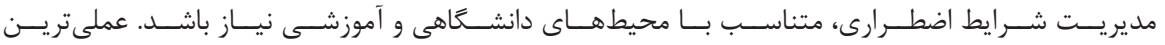

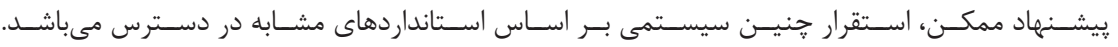

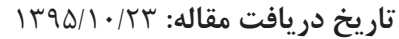

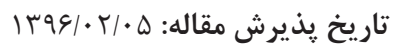

وازًَان كليدى: آمادَى در برابر شرايدى شرايط اضطرارى

دانشعاه

مميزى

همدامى حقوق نشر براى دانشكاه علوم يزشكى
فر كانسس بــروز شـرايط اضطــرارى از جملـه موضوعـات بحـث برانخَيز

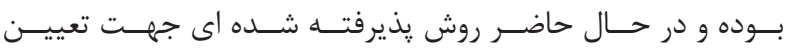

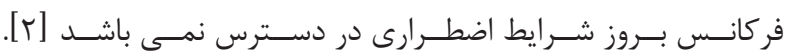

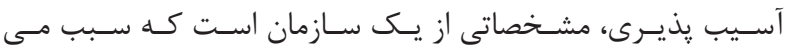

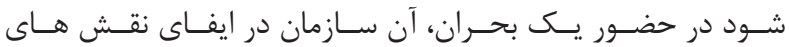

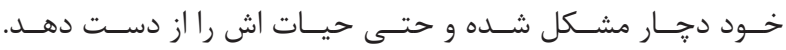

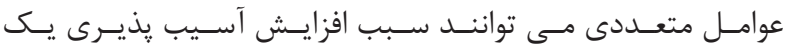

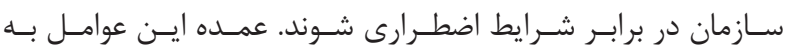

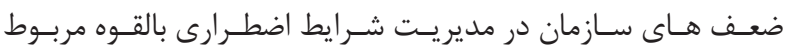

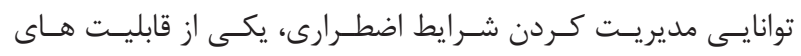

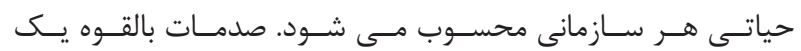

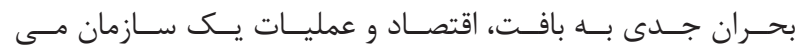

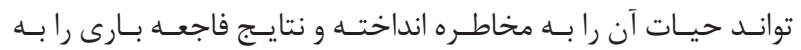

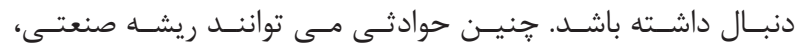

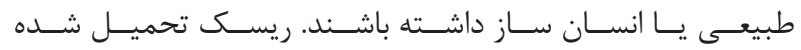

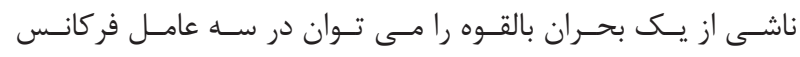

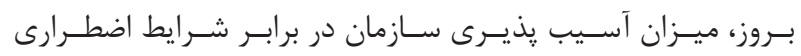

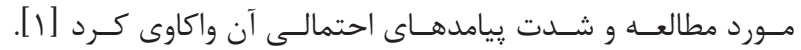


طـور تخصصى در سـطح جهـان فعاليـت مسى كنـــــ ايسن سـايت ها

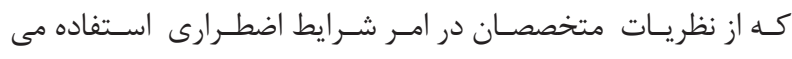

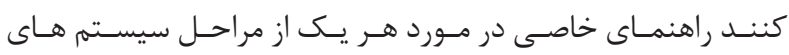

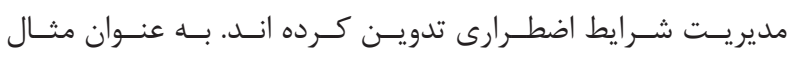
سـايت (ESA: Emergency and Safety Alliance) بـراى ايجـاد

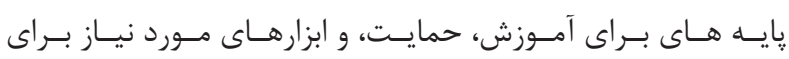

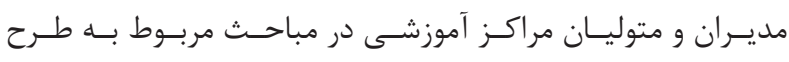

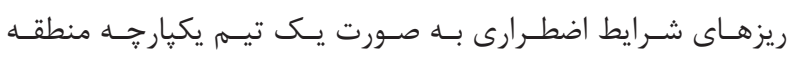

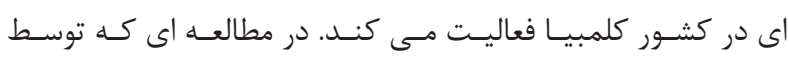

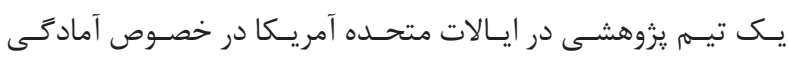

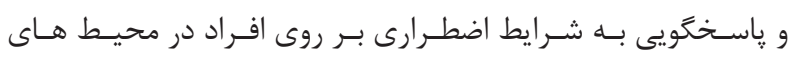

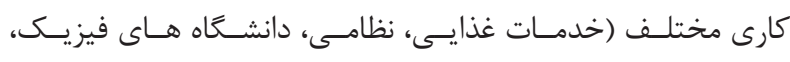

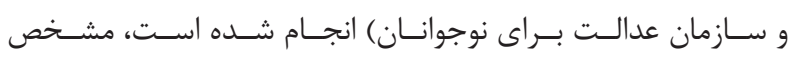

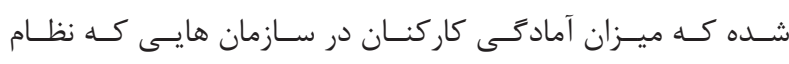

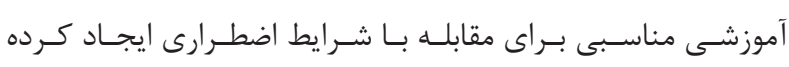

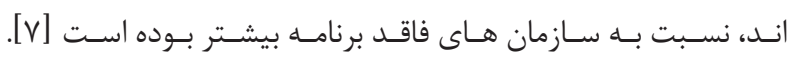

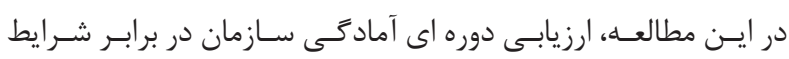

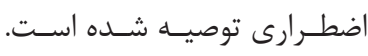

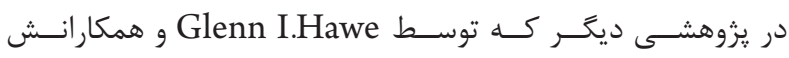

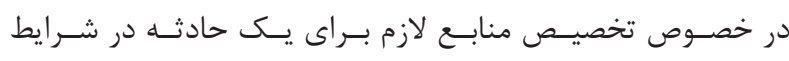

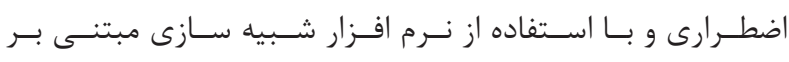

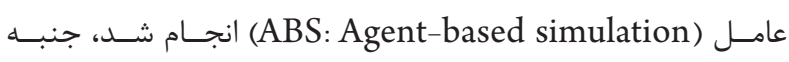

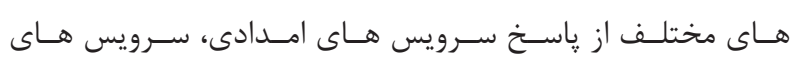

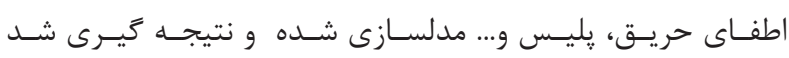

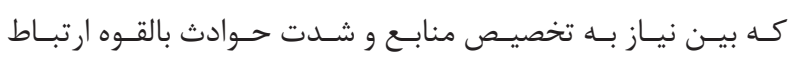

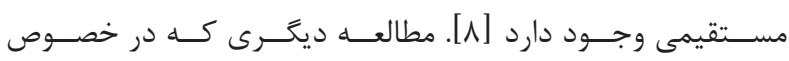

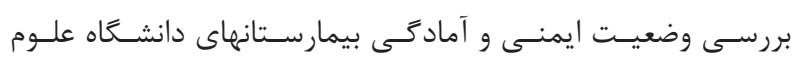

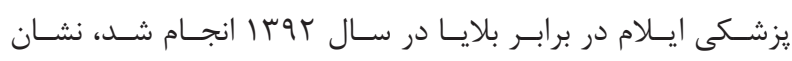

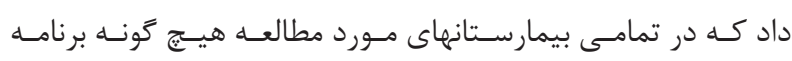

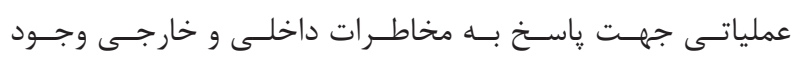

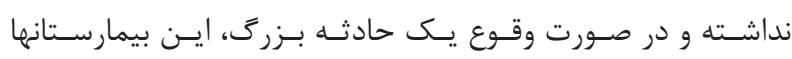

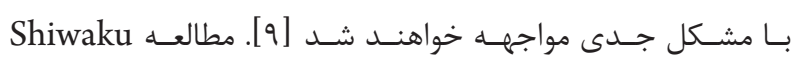

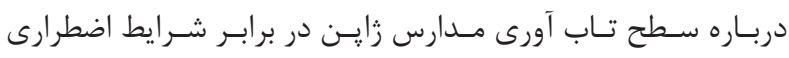

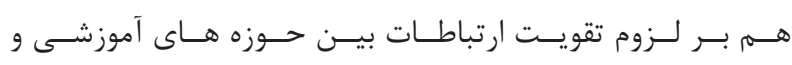

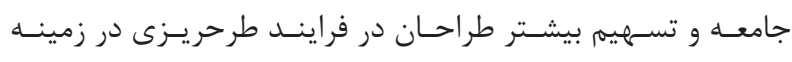

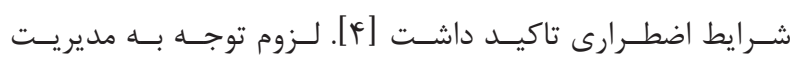

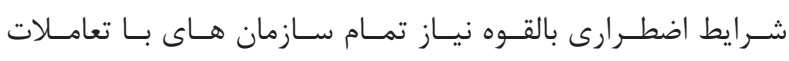

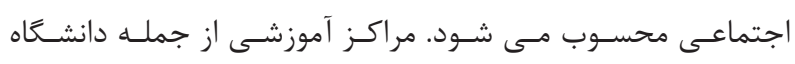

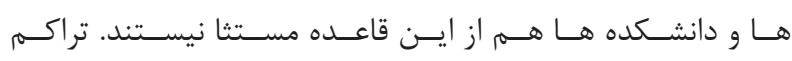

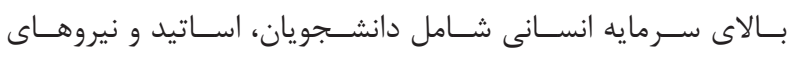

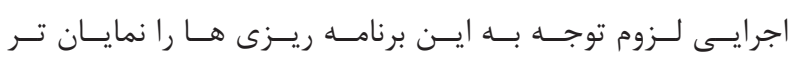

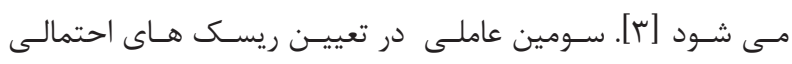

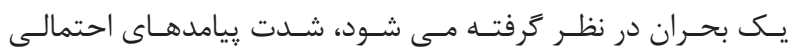

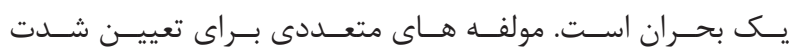

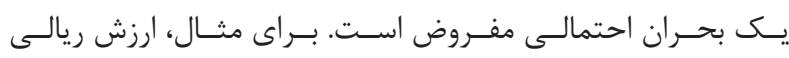

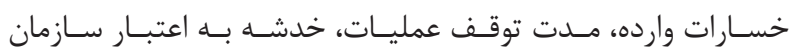

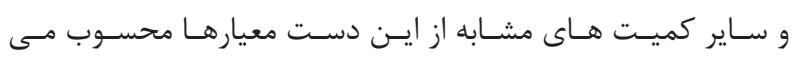

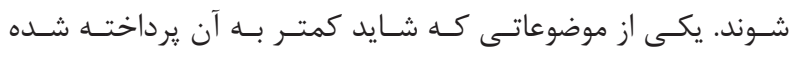

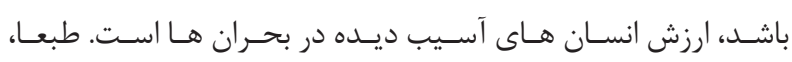

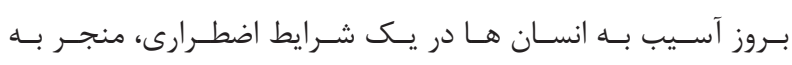

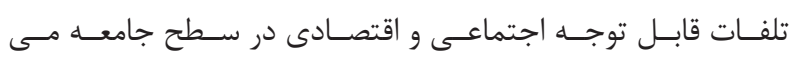

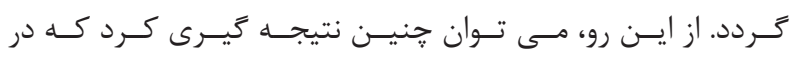

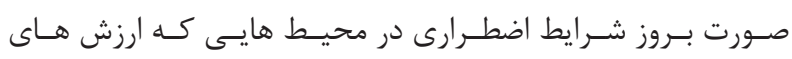

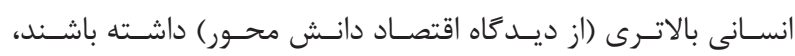

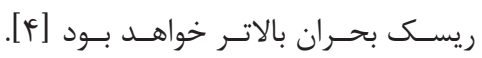

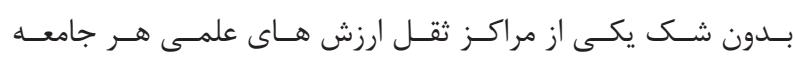

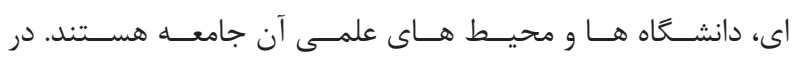

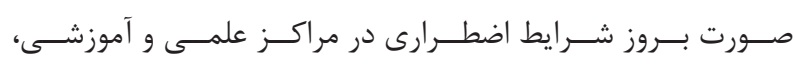

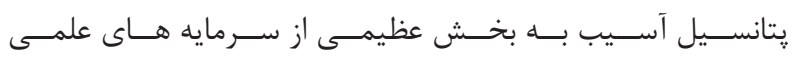

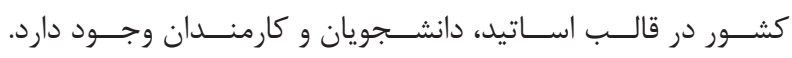

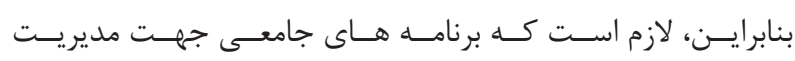

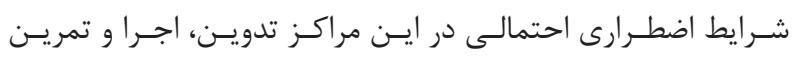

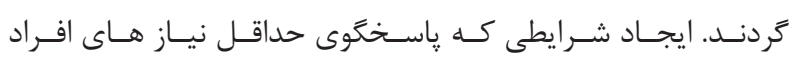

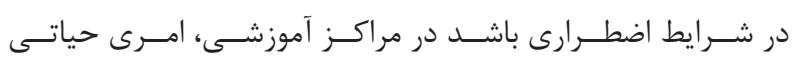

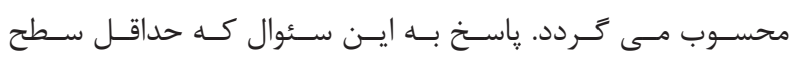

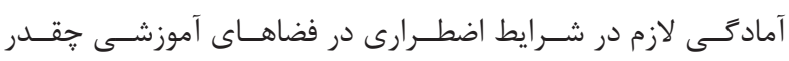

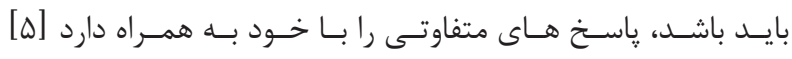

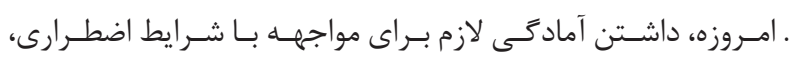

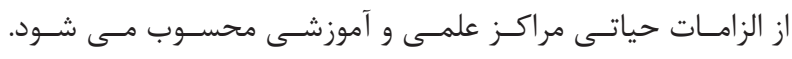

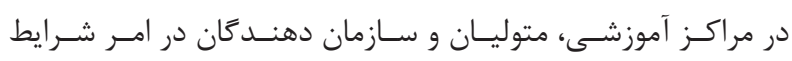

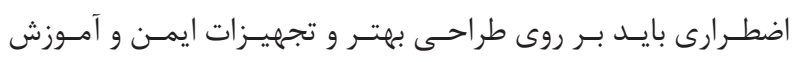

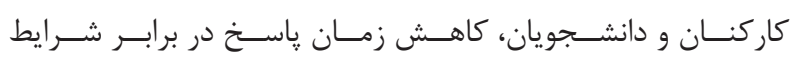

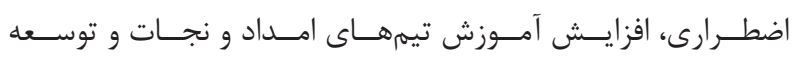

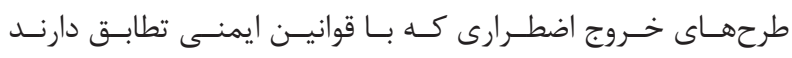

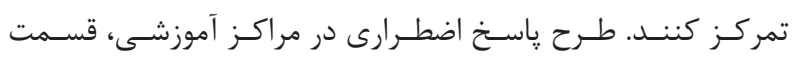

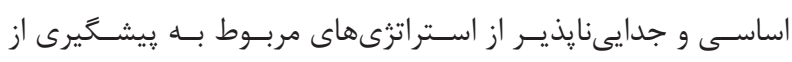

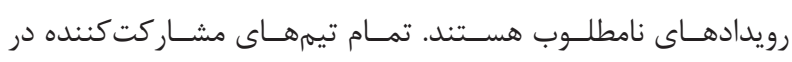

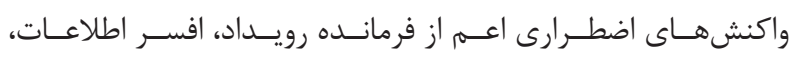

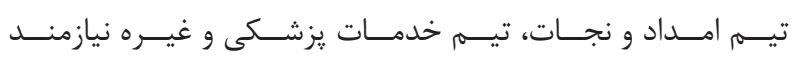

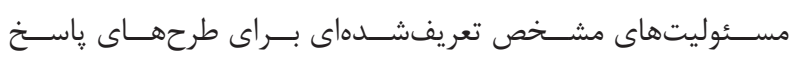

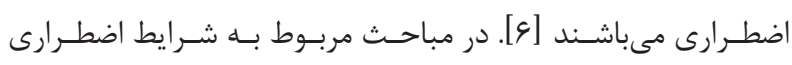

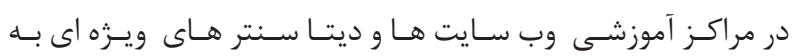




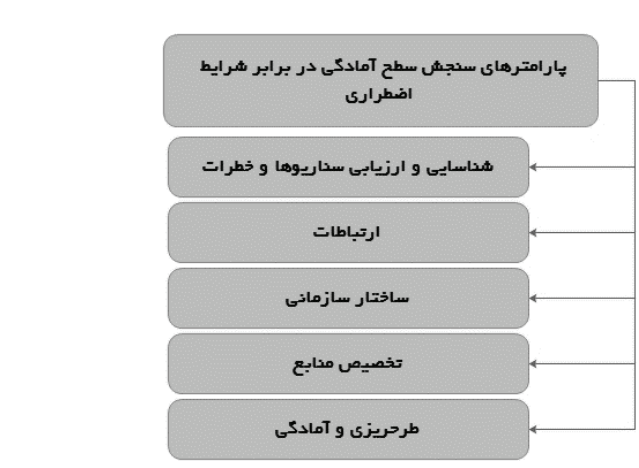

تصويـــر ا: ســرفصل هــاى اصلـى بررسـى ســـح آمادكَى در برابــر

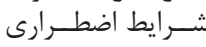

جهـت كمسى سـازى وضعيـت مـوارد بررسـى شـــه از يـك سيسـتم

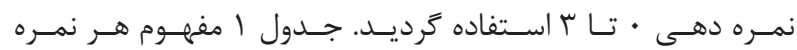

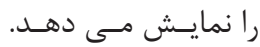

\begin{tabular}{|c|c|}
\hline \multicolumn{2}{|c|}{ 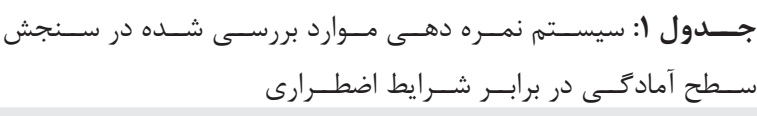 } \\
\hline تفسير & نمره \\
\hline عدم وجود آيتم مورد بررسى در سازمان & . \\
\hline آيتم مورد بررسى وجود دارد اما اجرا نشده يا اثربخش نيست. & 1 \\
\hline آيتهم مورد بررسى در سازمان وجود داشته، اجرا شده و تا استا & $r$ \\
\hline آيتم مورد بررسى در سازمان وجود داشته، اجرا شده و & r \\
\hline
\end{tabular}

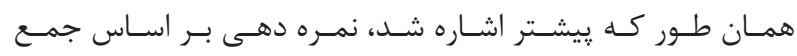
آورى شـواهد (مصاحبـه، مشـاهده، تسـت، نمونسه بــردارى و ...) انجام

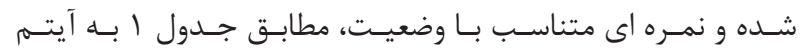

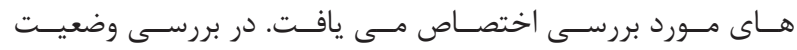

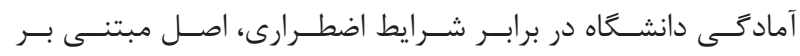

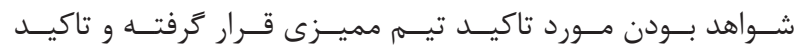

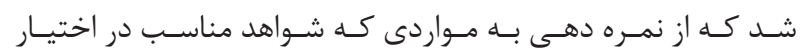

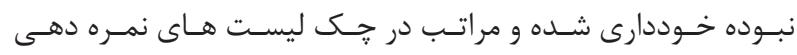

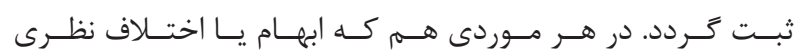

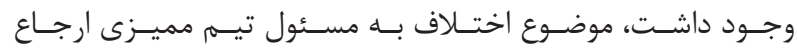

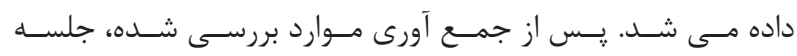

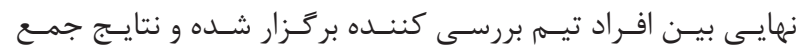

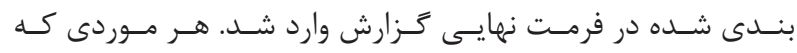

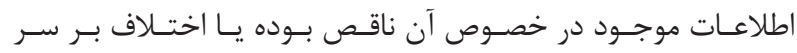

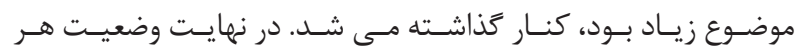

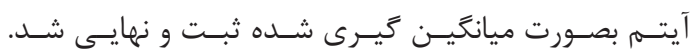

مــى نمايسـد. بــا توجــهـ بــه كمبــود مطالعــات حاضــر در زمينــهـ

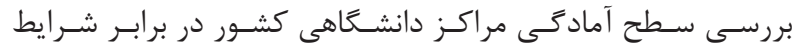

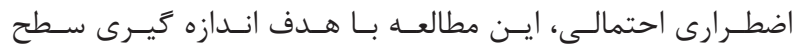

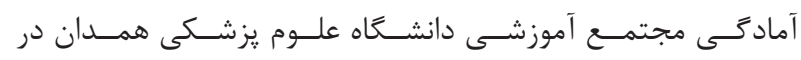

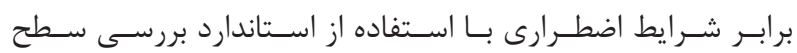

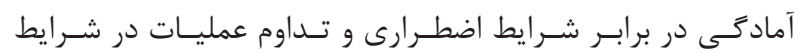

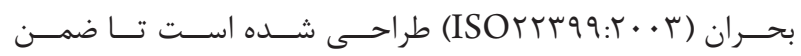

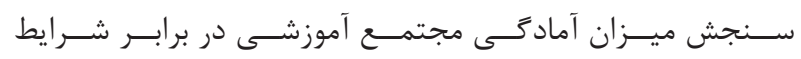

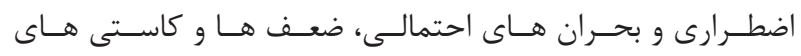

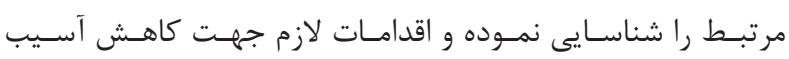

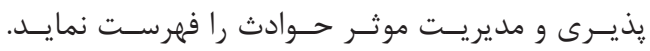

\section{روش كار}

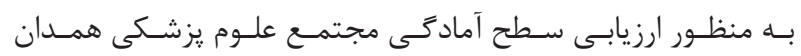

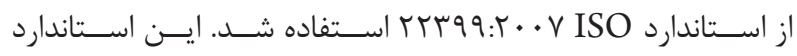

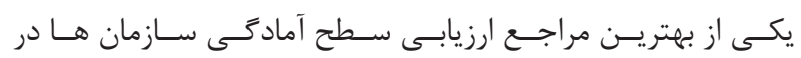

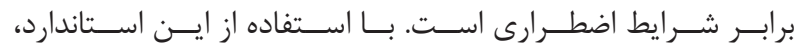

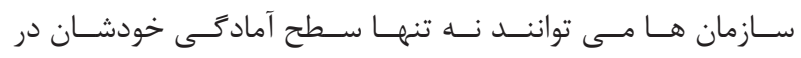

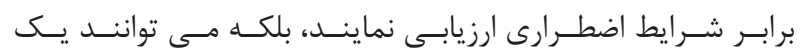

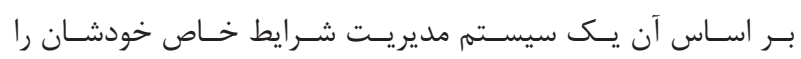

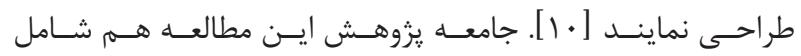

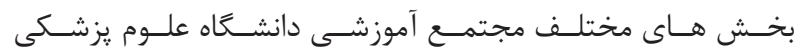

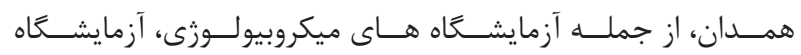

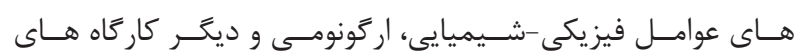

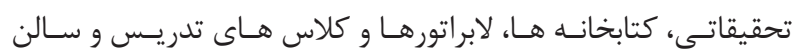

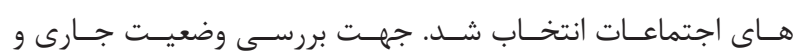

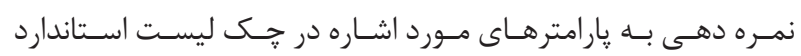

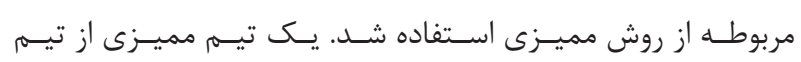

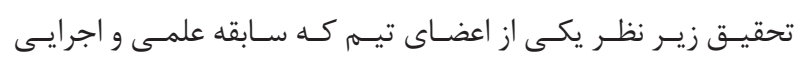

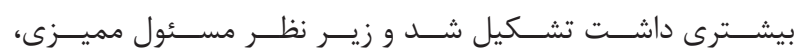

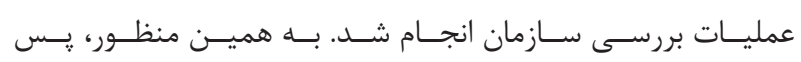

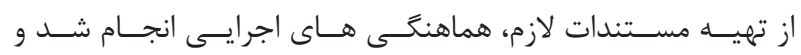

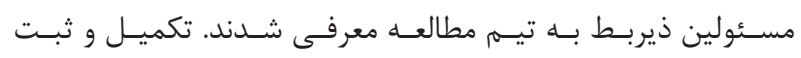

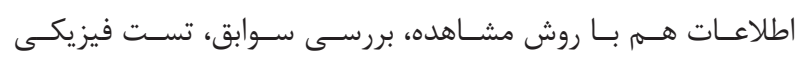

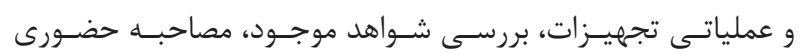

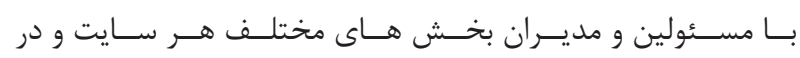

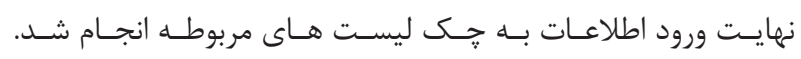

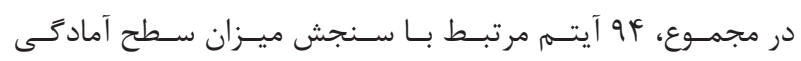

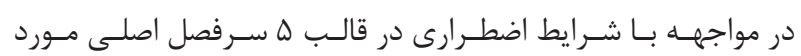

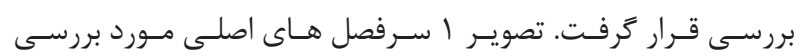

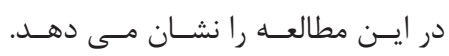


نمودار كلى ميزان آمادَى مجتمع آموزشى علوم يزشكى همدان در مواجهه با شرايط اضطرارى

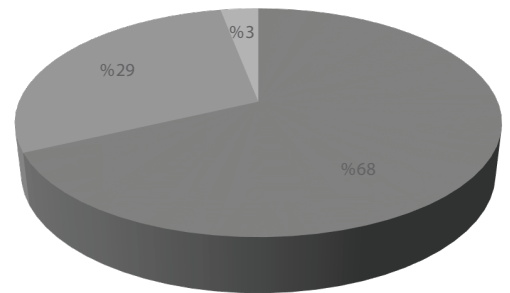

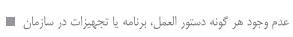

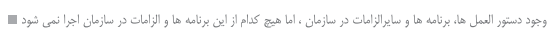

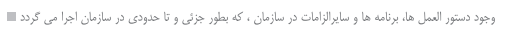

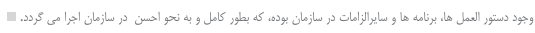

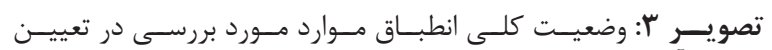

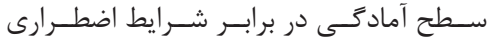

بحث

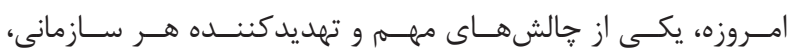

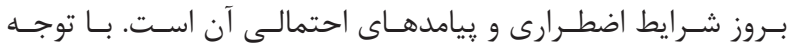

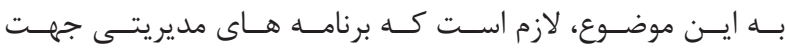

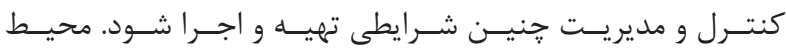

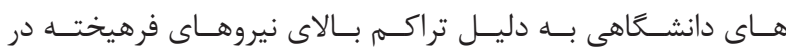

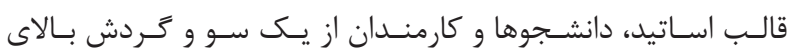

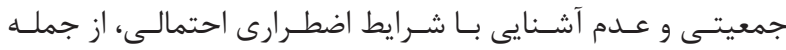

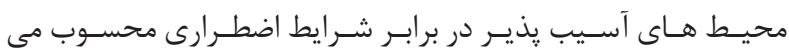

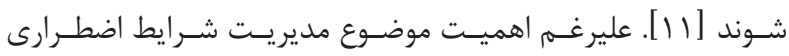

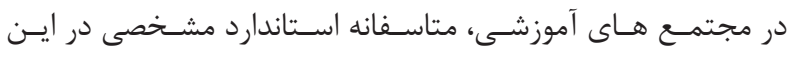

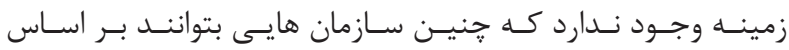

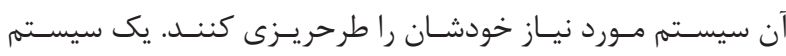

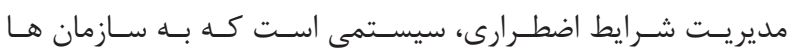

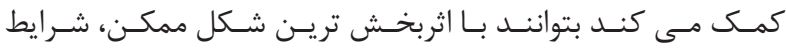

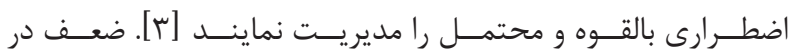

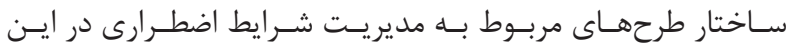

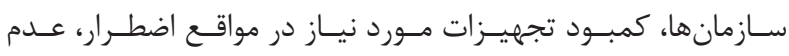

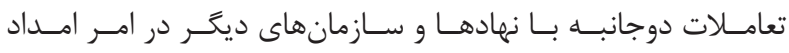

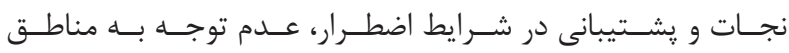

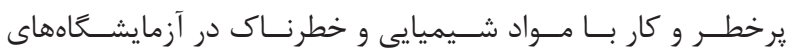

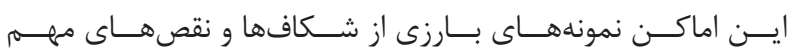

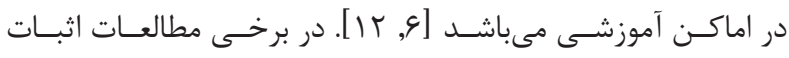

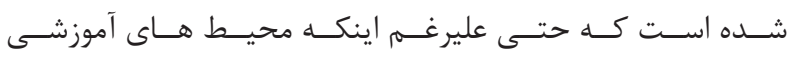

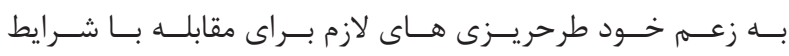

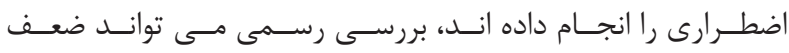

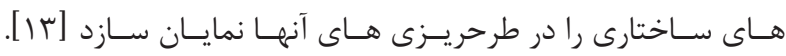

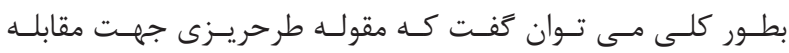

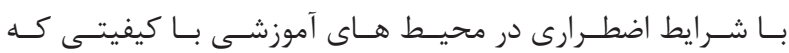

\section{بافته ها}

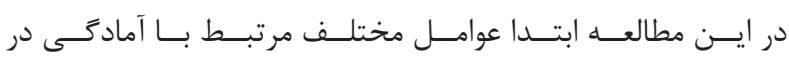

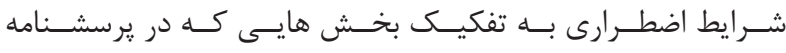

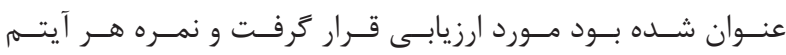

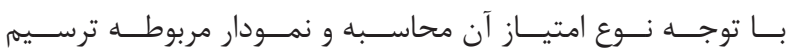

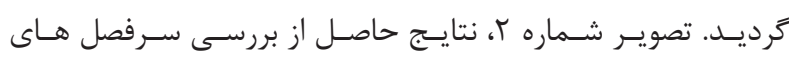

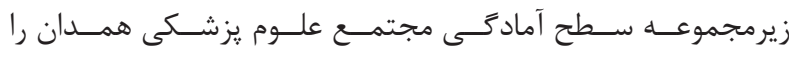

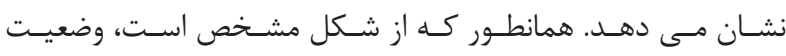

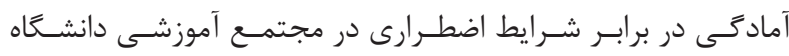

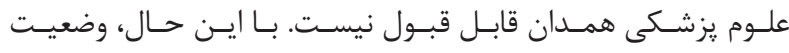

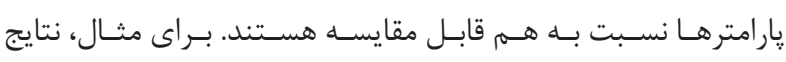

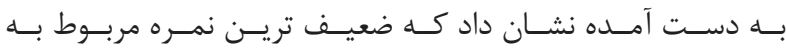

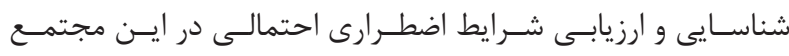

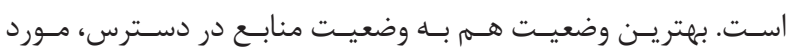

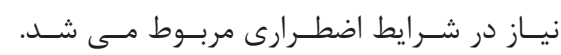

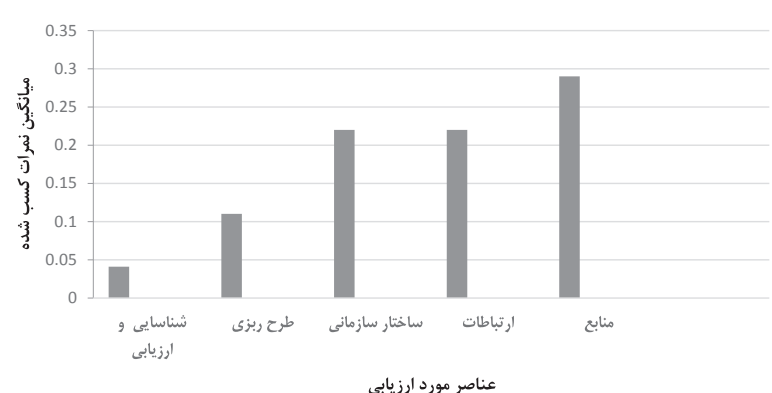

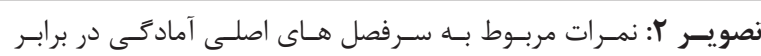

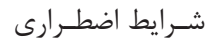

در مرحلـه دوم، امتيــازات كلـى عناصـر تمامسى بخـش هــــ مختلـف

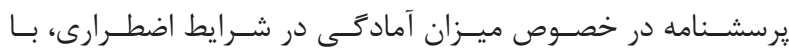

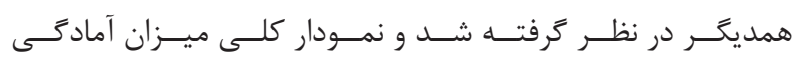

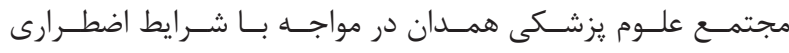

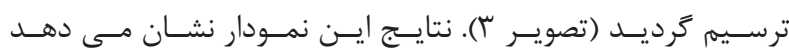

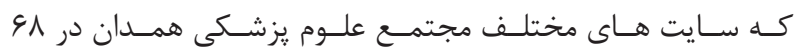

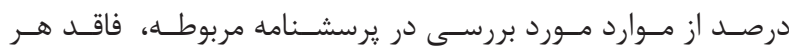

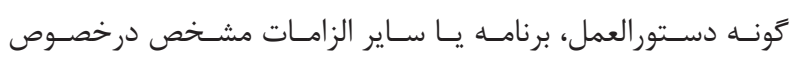

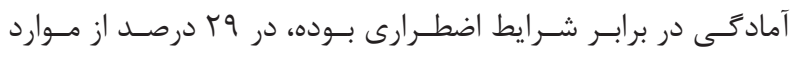

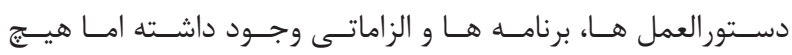

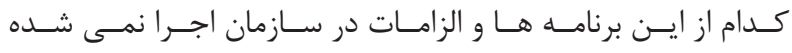

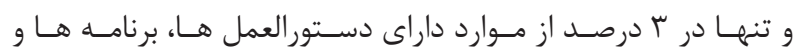

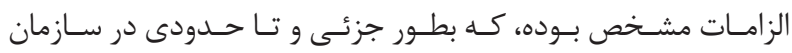
اجــرا مسى كردنـد. 


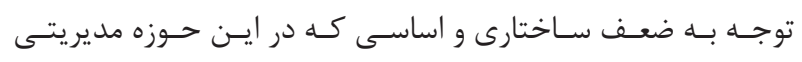

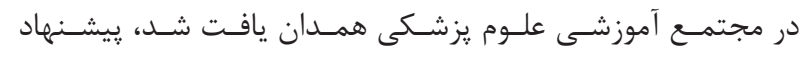

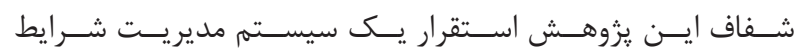

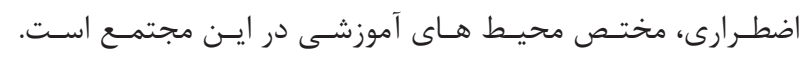

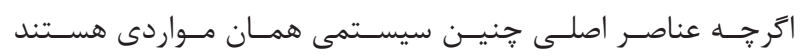

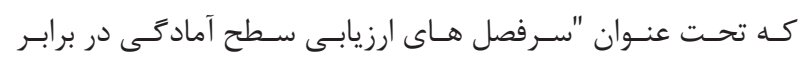

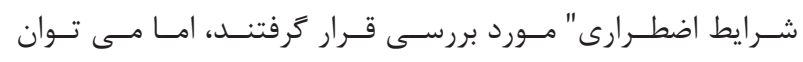

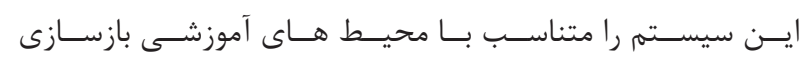

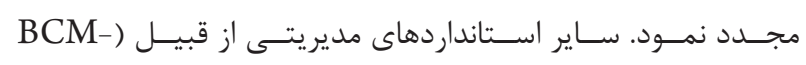
دارج (Business Continuity Management در ايـن مطالعـه از آن اسـتفاده گرديـد (ancident Preparedness (and Operational Continuity Management-IPOCM

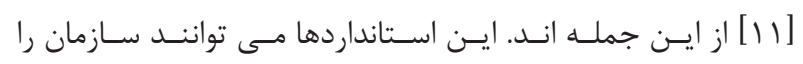

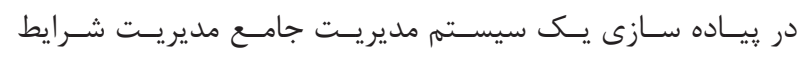

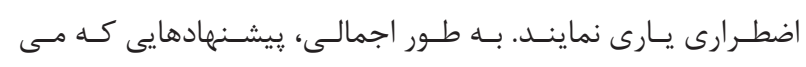

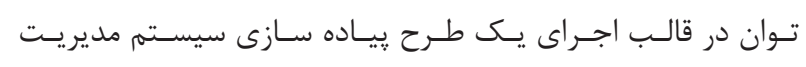

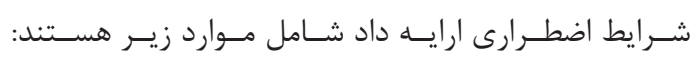

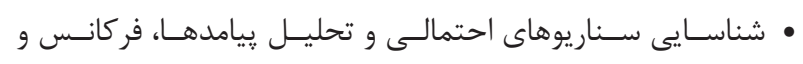

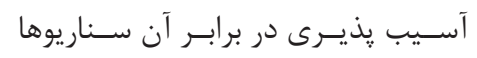

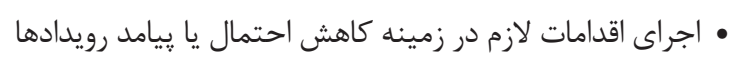

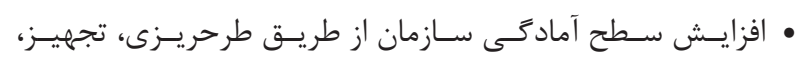

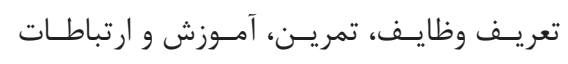

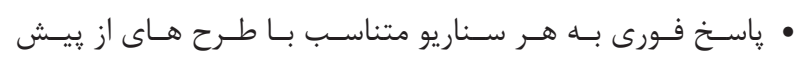
تدويسن شـده

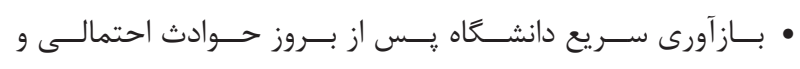

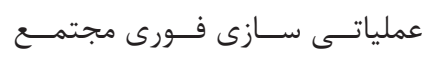

\section{REFERENCES}

1. Adini B, Ohana A, Furman E, Ringel R, Golan Y, Fleshler E, et al. Learning lessons in emergency management: the 4th International Conference on Healthcare System Preparedness and Response to Emergencies and Disasters. Disaster Mil Med. 2016;2:16. DOI: 10.1186/s40696-016-0026-3 PMID: 28265450

2. Yamamura E. Impact of natural disaster on public sector corruption. Public Choice. 2014;161(3-4):385-405. DOI: 10.1007/s11127-014$\underline{0154-6}$

3. Kalatpour O. Major accident prevention through applying safety knowledge management approach. J Emerg Manag. 2016;14(2):15360. DOI: $10.5055 /$ jem.2016.0281 PMID: 27108923

4. Shiwaku K, Ueda Y, Oikawa Y, Shaw R. School disaster resilience assessment in the affected areas of 2011 East Japan earthquake and tsunami. Nat Hazards. 2016;82(1):333-65. DOI: 10.1007/s11069-016-2204-5

5. Bobo N, Hallenbeck P, Robinson J, National Association of School N. Recommended minimal emergency equipment and resources for schools: national consensus group report. J Sch Nurs. 2003;19(3):150-6. DOI: 10.1177/10598405030190030501 PMID: $\underline{12755679}$

6. Sapien RE, Allen A. Emergency preparation in schools: a snapshot of a rural state. Pediatr Emerg Care. 2001;17(5):329-33. PMID: $\underline{11673708}$

7. Renschler LA, Terrigino EA, Azim S, Snider E, Rhodes DL, Cox CC. Employee Perceptions of Their Organization's Level of Emergency Preparedness Following a Brief Workplace Emergency Planning Ed-

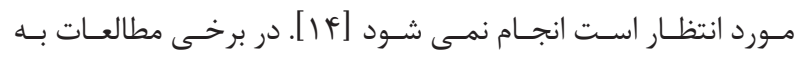

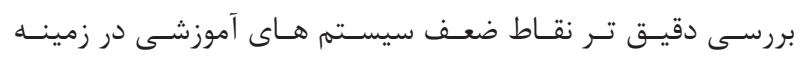

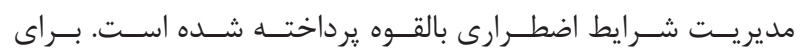

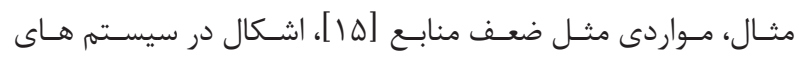

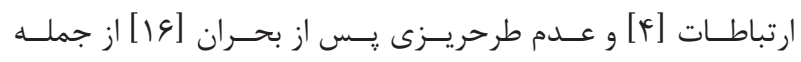

$$
\text { ايـن مـوارد محسـوب مـى شـوند. }
$$

\section{نتيجه تيرى}

نتايــج مطالعـه حاضــر نشـان داد كـهـ وضعيـت آمادكَى مجتمـع

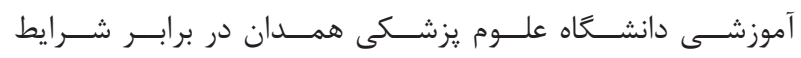

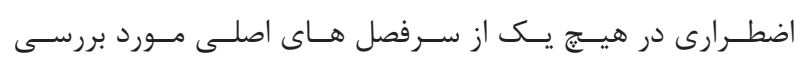

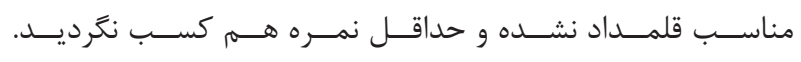

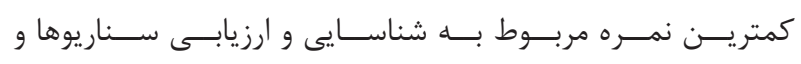

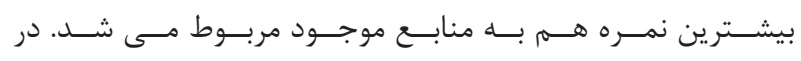

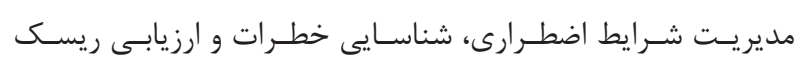

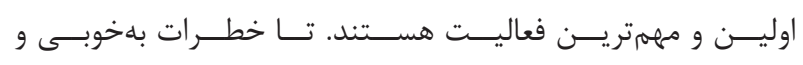

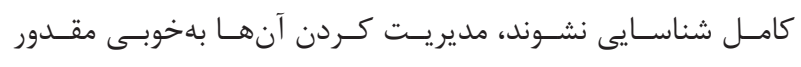

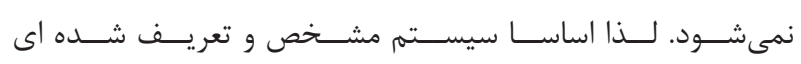

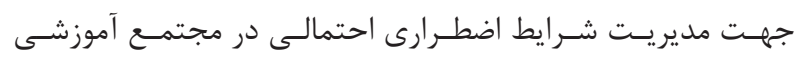

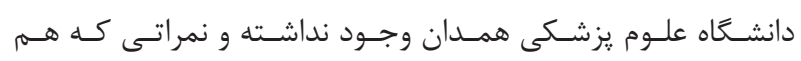
در بخـش هـاى مختلـف كسبـ شـده انـد، احتمـالا بـا سـاير مـوارد

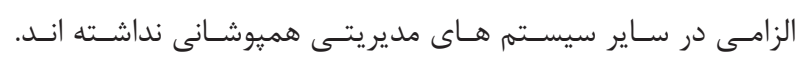

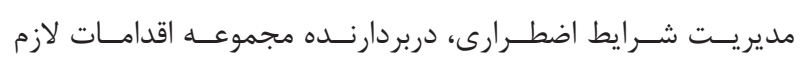

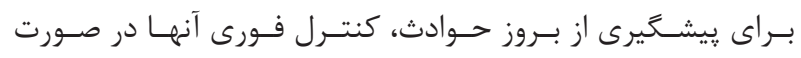

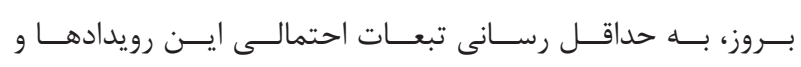

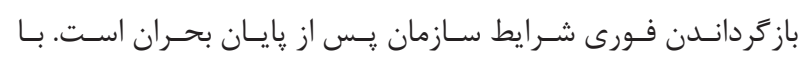

ucational Presentation. Saf Health Work. 2016;7(2):166-70. DOI: 10.1016/j.shaw.2015.10.001 PMID: 27340606

8. Hawe GI, Coates G, Wilson DT, Crouch RS. Agent-based simulation of emergency response to plan the allocation of resources for a hypothetical two-site major incident. Eng Appl Artif Intell. 2015;46:33645. DOI: $10.1016 /$ i.engappai.2015.06.023

9. Mirzaee F, Kakaei H, Farasati F, Zamani N. Investigation on the Safety Status and Preparedness of Ilam s Hospitals against Disasters in 2012. J Ilam Univ Med Sci. 2015;22(7):14-23.

10. Standardization IOf. Societal security - Guidelines for incident preparedness and operational continuity management. ISO; 2007.

11. Herbane B. The evolution of business continuity management: A historical review of practices and drivers. Busin Hist. 2010;52(6):9781002. DOI: $10.1080 / 00076791.2010 .511185$

12. Berkowitz Z, Haugh GS, Orr MF, Kaye WE. Releases of hazardous substances in schools: data from the Hazardous Substances Emergency Events Surveillance system, 1993-1998. J Environ Health. 2002;65(2):20-7, 37, 9. PMID: 12226905

13. Kano M, Ramirez M, Ybarra WJ, Frias G, Bourque LB. Are Schools Prepared for Emergencies? A Baseline Assessment of Emergency Preparedness at School Sites in Three Los Angeles County School Districts. Educ Urban Soc. 2007;39(3):399-422. DOI: $\underline{10.1177 / 0013124506298130}$

14. Klingman A. A school-based emergency crisis intervention in a mass school disaster. Prof Psychol Res Pract. 1987;18(6):604-12. DOI: 
10.1037/0735-7028.18.6.604

15. Stein CH, Vickio CJ, Fogo WR, Abraham KM. Making Connections: A Network Approach to University Disaster Preparedness. J Coll Stud Dev. 2007;48(3):331-43. DOI: 10.1353/csd.2007.0031

16. North CS, Kawasaki A, Spitznagel EL, Hong BA. The course of PTSD, major depression, substance abuse, and somatization after a natural disaster. J Nerv Ment Dis. 2004;192(12):823-9. PMID: 15583503

17. Gibb F, Buchanan S. A framework for business continuity management. Int J Inf Manag. 2006;26(2):128-41. DOI: 10.1016/j.ijinfomgt.2005.11.008 


\title{
Evaluation of the Emergency Preparedness Level at Training Complex of Hamadan University of Medical Sciences through Using ISO 22399:2003 and Suggestion of Improvement Measures
}

\author{
Ahmad Reza Gahar ${ }^{1}$, Hamed Takloo Bighash ${ }^{1}$, Safoura Karimi ${ }^{2}$, \\ Omid Kalatpour ${ }^{3, *}$ \\ ${ }^{1} M S c$, Department of Health Management, HSE Center, Faculty of Hygine, \\ Hamedan University of Medical Sciences, Hamedan, Iran \\ ${ }^{2}$ MSc, Department of Professional Health Engineering, Faculty of Hygine, Hamed- \\ an University of Medical Sciences, Hamedan, Iran \\ ${ }^{3}$ Assistant Professor, Department of Professional Health Engineering, Faculty of \\ Hygine, Hamedan University of Medical Sciences, Hamedan, Iran \\ ${ }^{*}$ Corresponding author: Omid Kalatpour, Assistant Professor, Department of Pro- \\ fessional Health Engineering, Faculty of Hygine, Hamedan University of Medical
}

DOI: $10.21859 /$ johe-03046

Received: $12 / 01 / 2017$

Accepted: 25/04/2017

\section{Keywords:}

Emergency Preparedness

University

Audit

How to Cite this Article:

Gahar AR, Takloo Bighash $\mathrm{H}$, Karimi S, KalatpourO. Evaluation of the Emergency Preparedness Level at Training Complex of Hamadan University of Medical Sciences through Using ISO 22399:2003 and Suggestion of Improvement Measures. $J \mathrm{Oc}$ cup Hyg. 2016;3(4):46-52. DOI: $10.21859 /$ johe- 03046

(C) 2016 Hamedan University of Medical Sciences.

\begin{abstract}
Introduction: Emergencies can impose huge tolls on the organizations. Such consequences, due to aggregation of the experts, could lead to the more catastrophic outcomes in the academic environments. Usually, the academic environments are less familiar with the management of the emergencies. The present paper aimed to measure the preparedness level against the emergencies in the Hamadan University of Medical Sciences.

Methods: In this study, a basic checklist was developed based on the ISO 22399:2003. Then, an audit team was established and conducted the audit process. Five core elements that were investigated included planning, organizational structure, resources, communication and scenario analysis. These items were scored 0-3 according to the sampling audit evidences.

Results: The overall status of all elements was assessed as "unacceptable". The least score belonged to the scenario analysis and the most was related to the resources.

Conclusions: Regarding the obtained results, it seems that there is a vital need to establish an emergency management system for Hamadan University of Medical Sciences. The most practical offer is following the accepted standards for implementing an emergency management system.
\end{abstract}

